\title{
Pulmonale Hypertonie: anästhesiologisches Management
}

Alexander Hötzel, Torsten Loop

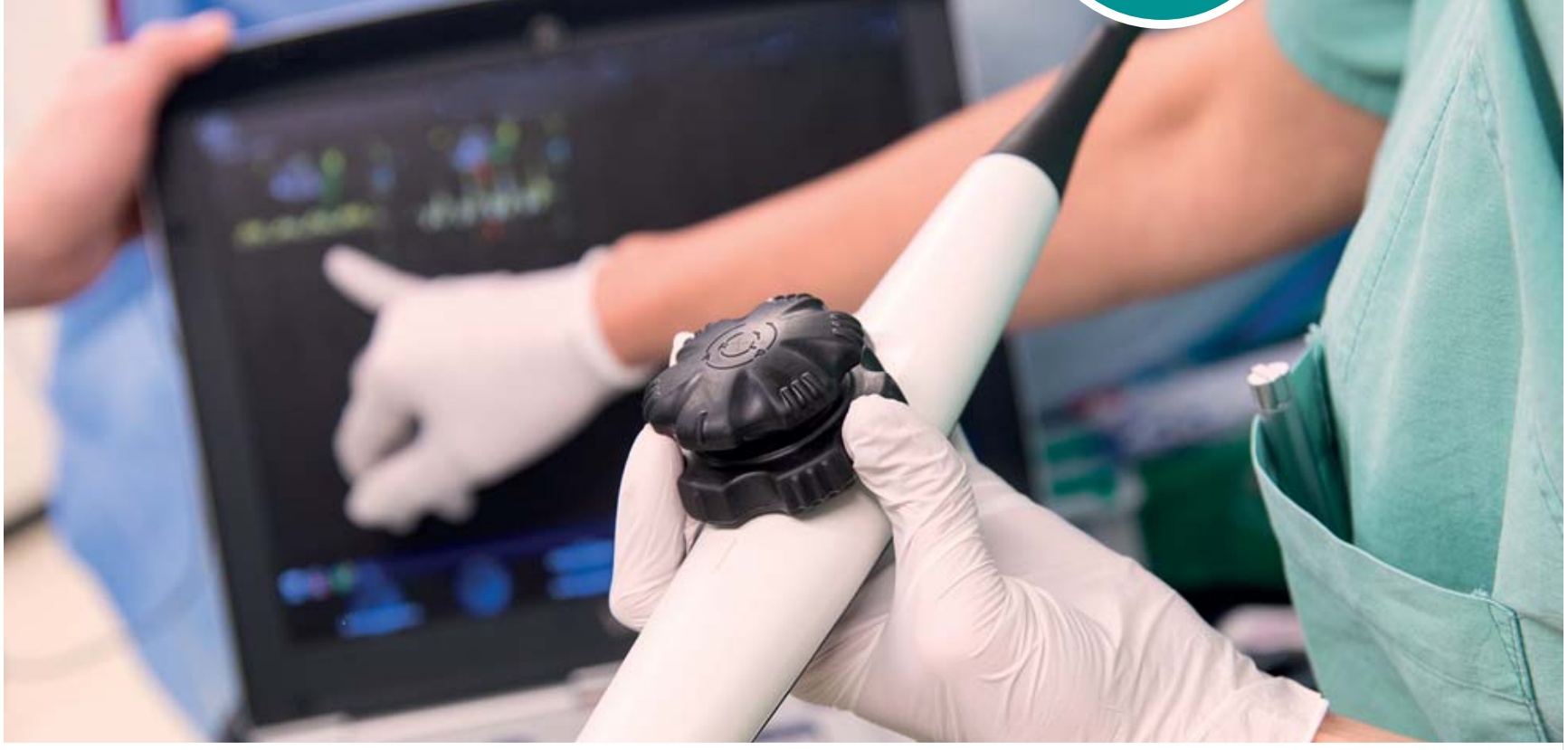

Quelle: KH Krauskopf.

Für das anästhesiologische perioperative Management von Patienten mit pulmonaler Hypertonie sind fundierte Kenntnisse der Erkrankung essenziell - nur so lässt sich eine sichere Versorgung dieser Patienten gewährleisten. Das Ziel der Übersicht ist es, insbesondere Kollegen, die nicht täglich in der Herz-/Thoraxanästhesie tätig sind, das notwendige anästhesiologische Wissen zu vermitteln.

\section{Einleitung}

Bezüglich detaillierter Darstellungen der Grundlagen zur pulmonalen Hypertonie (PH) möchten wir auf den Artikel von Lücke et al. in dieser Ausgabe verweisen [1]. Erforderlich für das anästhesiologische perioperative Management der Patienten mit PH sind insbesondere Kenntnisse der

- Ursachen,

- Pathophysiologie,

- vorausgehenden Behandlung,

- perioperativen Optionen des Monitorings als auch

- therapeutischer Interventionsmöglichkeiten.

\section{Präoperative Evaluation}

Eine bislang nicht diagnostizierte $\mathrm{PH}$ bei der präoperativen Evaluation eines Patienten zu detektieren, erscheint aufgrund der unspezifischen klinischen Symptome schwierig.

\begin{abstract}
Merke
In der Vorbereitung der Patienten mit bekannter $\mathrm{PH}$ sind insbesondere die Kenntnis der Ursache(n), der Schweregrad der Erkrankung und die Rechtsherzfunktion von entscheidender Bedeutung.
\end{abstract}

\section{Ursache der pulmonalen Hypertonie}

Meist präsentieren sich die Patienten mit bekannter $\mathrm{PH}$ mit entsprechenden Vorbefunden. Als Standarduntersuchung zur Verifizierung einer $\mathrm{PH}$ gilt nach wie vor die 
- Tab. 1 Ursachen der prä-/postkapillären pulmonalen Hypertonie [3].

\begin{tabular}{|l|l|l|}
\hline Definition der PH & Grenzwerte & Beispiele zugrunde liegender Ursachen \\
\hline präkapillär & PAPm $\geq 25 \mathrm{mmHg}$ & - idiopathische pulmonalarterielle Hypertonie \\
\hline PCWP $\leq 15 \mathrm{mmHg}$ & $\begin{array}{l}\text { " PH durch chronische Lungenerkrankung } \\
\text { - chronisch thromboembolische PH }\end{array}$ \\
\hline postkapillär & \begin{tabular}{l} 
" PH durch systolische oder diastolische Linksherzdysfunktion, Vitien \\
\hline
\end{tabular} & \\
\hline PAPm $\geq 25 \mathrm{mmHg}$ & \\
\hline
\end{tabular}

PH: pulmonale Hypertonie, PAPm: mittlerer pumonalarterieller Druck, PCWP: pulmonalkapillärer Verschlussdruck

- Tab. 2 Funktionelle Klassifikation der pulmonalen Hypertonie [3, 6].

\begin{tabular}{|c|c|}
\hline Klasse & Symptomatik der Patienten mit nachgewiesener pulmonaler Hypertonie \\
\hline 1 & ohne Einschränkung der körperlichen Aktivität \\
\hline 2 & leichte Einschränkung der körperlichen Aktivität ohne Beschwerden in Ruhe \\
\hline 3 & $\begin{array}{l}\text { deutliche Einschränkung der körperlichen Aktivität ohne Beschwerden in Ruhe; bereits leichtere als normale Belastungen } \\
\text { führen zu Dyspnoe oder Müdigkeit, thorakalen Schmerzen oder Schwächeanfällen }\end{array}$ \\
\hline 4 & $\begin{array}{l}\text { keinerlei körperliche Belastung ohne Beschwerden möglich und/oder bereits in Ruhe vorhandene Beschwerden; Zeichen der } \\
\text { manifesten Rechtsherzinsuffizienz }\end{array}$ \\
\hline
\end{tabular}

Rechtsherzkatheter-Untersuchung [2]. Hieraus lässt sich die Frage beantworten, ob eine präkapilläre, postkapilläre, oder gemischte Form der PH vorliegt $(\triangleright$ Tab. 1$)$. Diese Unterscheidung lässt Rückschlüsse auf die Ätiologie der $\mathrm{PH}$ zu, erklärt pathophysiologische Zusammenhänge und steuert somit in der perioperativen Versorgung die unterstützende Therapie.

Bei der Rechtsherzkatheter-Untersuchung wird oftmals die potenzielle Therapierbarkeit mittels kurzwirksamer pulmonaler Vasodilatatoren überprüft. Sie gibt dem Anästhesisten wertvolle Informationen zur perioperativen Pharmakotherapie [4]. In der Mehrzahl der Fälle zeigt sich die $\mathrm{PH}$ als Symptom aufgrund unterschiedlicher Grunderkrankungen und nicht als eigenständige Erkrankung. Daher liegen bei der präoperativen Evaluation häufig ergänzende Untersuchungen zur Lungenfunktion, zum Ventilation-Perfusions-Verhältnis, Gefäßstatus, Herzfunktion etc. vor, die von dem verantwortlichen Anästhesisten objektiviert werden müssen.

\section{Schwere der pulmonalen Hypertonie}

Die Schweregradeinteilung erfolgt entsprechend dem gemessenen pulmonalarteriellen Mitteldruck (PAPm) in milde bis schwere Formen der PH. Darüber hinaus ist bei der präoperativen Evaluation vor allem die Belastbarkeit des Patienten zu eruieren [5]. Klinisch lässt sich die Erkrankungsschwere in Anlehnung an die funktionelle Klassifikation der Herzinsuffizienz der WHO einschätzen ( $\vee$ Tab. 2).

\section{Rechtsherzfunktion}

Der chronisch erhöhte pulmonal-vaskuläre Widerstand (pulmonary vascular resistance, PVR) führt zu einer kon- tinuierlichen Druckbelastung des rechten Ventrikels. Sowohl die rechtsventrikuläre Wanddicke als auch der Durchmesser nehmen zu. Die Rechtsherzhypertrophie und -dilatation mit deren Ausmaß sowie ein vorhandener Perikarderguss lassen sich in der Echokardiografie gut darstellen und abschätzen ( $\triangleright$ Abb. 1) [7]. Des Weiteren bietet die Echokardiografie die Möglichkeit, die Vorwölbung des Septums nach links und die konsekutive Reduktion der linksventrikulären Füllung zu visualisieren [8].

Die echokardiografische Einschätzung dieser Faktoren erscheint wichtig, da eine Rechtsherzhypertrophie, -dilatation und verminderte linkventrikuläre Füllung die nicht unerhebliche Gefahr bergen, intraoperativ die rechtskoronare Perfusion einzuschränken. Als Konsequenz muss bei dieser Konstellation intraoperativ mit einer Ischämie im rechten Herzen und einem Rechtsherzversagen gerechnet werden. Wurde eine Rechtsherzkatheter-Untersuchung durchgeführt, ergeben sich hieraus Hinweise auf die rechtsventrikuläre Funktion:

\section{Merke diale Funktion hin [9]. \\ Zusätzliche Untersuchungen und perioperatives Risiko}

Insbesondere ein niedriger rechtsatrialer Druck, ein hoher Herzindex und eine normale gemischtvenöse Sauerstoffsättigung deuten auf eine gute rechtskar-

Ein Elektrokardiogramm kann in Kombination mit der Echokardiografie bei Patienten mit PH wertvolle Informationen liefern [3]. Weitergehende Untersuchungen, wie die Bestimmung von ProBNP, ein Röntgenthorax oder 


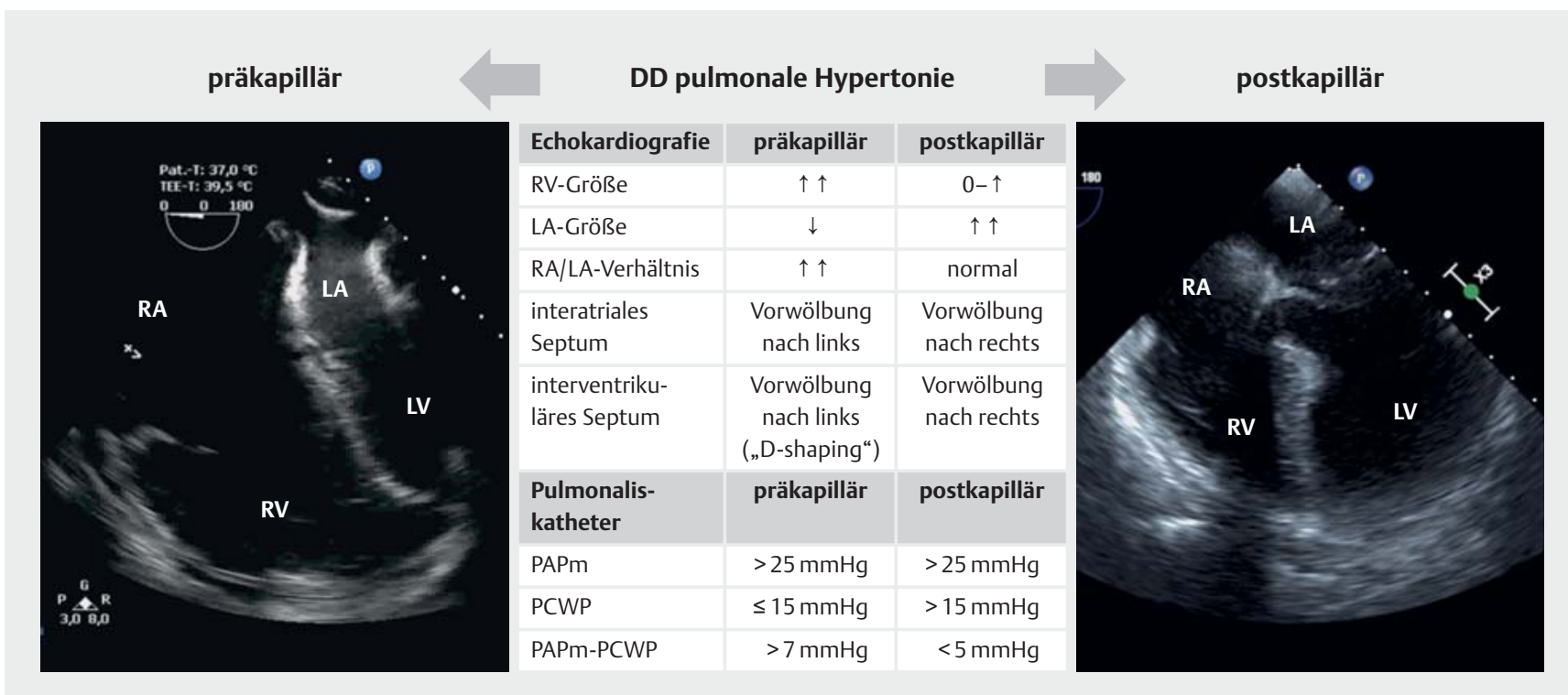

- Abb. 1 Vereinfachte echokardiografische und pulmonalarterielle Parameter zur Differenzialdiagnose einer prä- und postkapillären pulmonalen Hypertonie. Dargestellt sind Schnitte der transösophagealen Echokardiografie (s. auch \ Video 1 [präkapillär] und \Video 2 [postkapillär]). RA: rechter Vorhof, RV: rechter Ventrikel, LA: linker Vorhof, LV: linker Ventrikel, PAPm: mittlerer pulmonalarterieller Druck, PCWP: pulmonalkapillärer Verschlussdruck, $\uparrow:$ Zunahme, 0: kein wesentlicher Einfluss, $\downarrow:$ Abnahme.

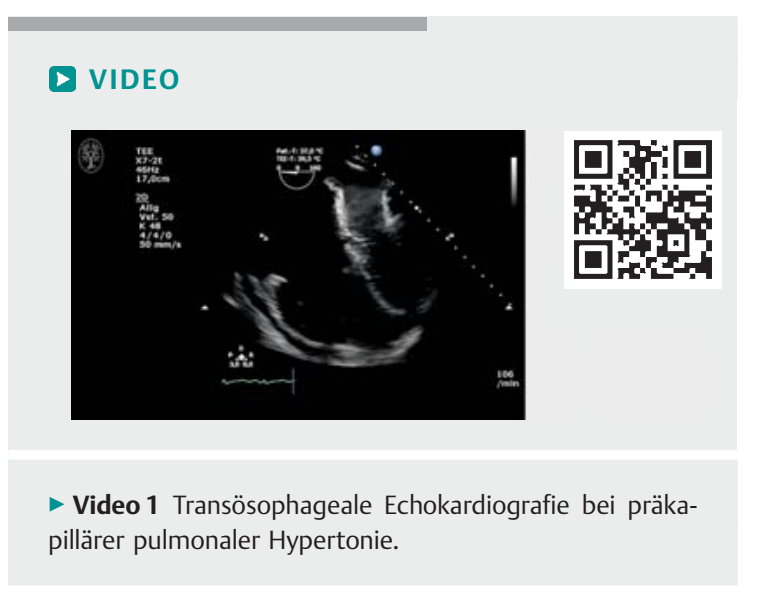

eine erneute Rechtsherzkatheter-Untersuchung, erscheinen vor den diagnostischen Möglichkeiten einer Echokardiografie und der meist fehlenden Konsequenz wenig sinnvoll.

Das perioperative Risiko ergibt sich aus der Kombination von Schweregrad der PH und Art des chirurgischen Eingriffs. Patienten in der funktionellen Klasse 1 können in aller Regel problemlos zu elektiven Operationen zugelassen werden [9]. Ein ähnliches Risiko weisen Patienten mit milder Symptomatik und geringem operativem Risiko auf. Die perioperative Morbidität und Letalität steigen hingegen bei erheblicher klinischer Einschränkung in Kombination mit großen Operationen oder Notfalleingriffen erheblich an [10]. Patienten in der funktionellen Klasse 4 müssen als Hochrisikopatienten eingestuft und

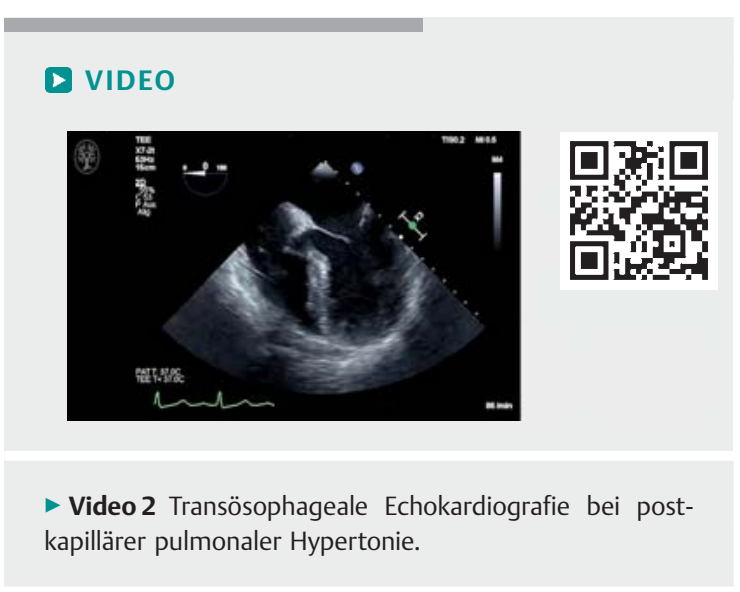

nicht essenzielle Operationen kritisch überdacht werden [11]. Bei den perioperativen Komplikationen ist die Rechtsherzdekompensation mit bis zur Hälfte aller Ursachen für die Letalität dieser Patienten verantwortlich [12]. Eine allgemeingültige perioperative Risikostratifizierung für Patienten mit PH ist kaum möglich.

\section{Merke}

Es gilt, das patientenbezogene Risiko mit dem zu erwartenden operativen Risiko individuell abzuwägen und mit dem Patienten und dem chirurgischen Partner zu besprechen.

\section{Prämedikation}

Eine anxiolytische Prämedikation bei symptomatischen $\mathrm{PH}-$ Patienten sollte - wenn überhaupt - nur mit äußers- 
> Tab. 3 Einfluss intravenöser und inhalativer Anästhetika auf den pulmonal-vaskulären Widerstand und die rechtsventrikuläre Kontraktilität [10].

\begin{tabular}{|l|l|l|l|l|l|l|l|l|}
\hline Anästhetika & Opioide & Etomidat & Propofol & Barbiturat & Ketamin & $\mathbf{N}_{2} \mathrm{O}$ & $\begin{array}{c}\text { Isofluran } \\
\text { Desfluran }\end{array}$ & \begin{tabular}{c} 
Sevofluran \\
\hline PVR
\end{tabular} \\
\hline Kontraktilität rechtes Herz & 0 & 0 & $\downarrow$ & 0 & $0-\uparrow$ & $\uparrow \uparrow$ & $\uparrow$ \\
\hline PVR: pulmonal-vaskulärer Widerstand, $\uparrow:$ Zunahme, 0: kein wesentlicher Einfluss, $\downarrow$ : Abnahme & $\downarrow$ & $\downarrow$ \\
\hline
\end{tabular}

ter Vorsicht und unter Monitoring erfolgen. Eine dadurch ausgelöste Hypoventilation mit Hypoxie und Hyperkapnie würde die PH aggravieren. Die vorbestehende Dauermedikation mit pulmonalen Dilatatoren sollte obligat weitergeführt werden, um einen unmittelbar perioperativen Rebound-Effekt zu vermeiden. Besteht die Gefahr, dass die intestinale Resorption dieser Medikamente gestört ist, kann die Therapie unter Einbeziehung der behandelnden Pulmologen/Kardiologen auf eine inhalative oder i. v. Applikationsform umgestellt werden.

\section{Narkoseeinleitung}

\section{Anästhesieverfahren}

Klare Vorteile bestimmter Anästhesieverfahren sind aufgrund der aktuell begrenzten Studien- und Datenlage nicht dokumentiert. Wichtig für die Narkoseeinleitung erscheint unter pathophysiologischen Gesichtspunkten eine „stressfreie“ Situation, da eine sympathische Stimulation die rechtsventrikuläre Nachlast erhöhen kann. Die Antizipation der zu erwartenden Nebenwirkungen der genutzten Medikamente erfordert eine „vorausschauend-kompensierende“ Arbeitsweise.

\section{Regionalanästhesie}

Regionalanästhesiologische Verfahren können unter Beachtung der eventuell bestehenden Antikoagulation durchgeführt werden. Gleiches gilt für lumbale neuroaxiale Verfahren $[3,13]$. Der Einsatz einer thorakalen Epiduralanästhesie bei Patienten mit schwerer $\mathrm{PH}$ bleibt in Diskussion, ohne dass eindeutige Daten den Stellenwert definieren würden. Nach eigenen Erfahrungen ist die hierdurch verbesserte postoperative Analgesie, die Einsparung atemdepressiver Opioide und die damit verbundene Normoventilation bei diesen Patienten ausgesprochen günstig. Die mit der Sympathikolyse assoziierte systemische Hypotension mit der potenziell verminderten Koronarperfusion und reduziertem kardialem Auswurf bei thorakaler Epiduralanästhesie muss jedoch vermieden werden $[13,14]$.

Eine die Regionalanästhesie begleitende Sedierung oder alleinige Analgosedierung ist bei Patienten mit symptomatischer PH nur mit allergrößter Vorsicht durchzuführen. Da Sauerstoff die pulmonalen Gefäße dilatiert und
Kohlendioxid den pulmonal-vaskulären Widerstand erhöht, gilt es Hypoxie und Hyperkapnie zu vermeiden.

Merke
Es empfiehlt sich, vor Beginn der Sedierung Sauer-
stoff via Maske oder Nasenbrille zu applizieren und
die Spontanatmung mittels Atemfrequenz und Kap-
nografie zu überwachen.

\section{Allgemeinanästhesie}

Bei der Wahl der Medikamente zur Einleitung einer Allgemeinanästhesie sind deren Wirkungen auf den pulmonalen Gefäßwiderstand und die myokardiale Kontraktilität zu berücksichtigen ( $\bullet$ Tab. $\mathbf{3}$ ).

Ein besonders nebenwirkungsarmes Profil besitzen Opioide wie Fentanyl oder Sufentanil [15]. Remifentanil wirkt zudem histaminvermittelt gering vasodilatierend [16]. In der Literatur wird oftmals Etomidat aufgrund der geringen Beeinflussung der systemischen und pulmonalen Zirkulation als besonders günstig bei PH und/oder Rechtsherzdysfunktion postuliert $[10,17]$. Nach eigener Erfahrung lässt sich auch Propofol einsetzen. Es zeigt zwar keine wesentlichen Effekte auf die pulmonalen Gefäße, kann jedoch den systemischen Widerstand und die rechtsventrikuläre Kontraktilität beeinträchtigen.

Merke
Bei der Induktion einer Allgemeinanästhesie er-
scheint es den Autoren besonders wichtig, auf den
prophylaktischen Einsatz vasokonstringierender
Substanzen hinzuweisen, um die zu antizipierende
Hypotension bei Einleitung zu verhindern.

\section{Auswahl des Monitorings}

Datenbasierte Empfehlungen zu Art, Ausmaß und Einsatzkriterien eines invasiven Monitorings bei Patienten mit PH fehlen gänzlich [10]. Deshalb sind die Erfahrung und Einschätzung des behandelnden Anästhesisten entscheidend - auf der Basis der patientenbezogenen Evaluation und der operativen Risiken. Kleinere Eingriffe bei Patienten der funktionellen Klasse 1-2 sollten unter Standardmonitoring durchführbar sein. Anders ist es bei zunehmender Erkrankungsschwere mit erheblicher Beeinträchtigung der funktionellen Kapazität: Hier empfiehlt sich nach Ansicht der Autoren die Etablierung einer inva- 
siven Blutdruckmessung, gefolgt von einer Blutgasanalyse vor der Narkoseeinleitung. Vor allem in der Kombination mit zu erwartenden Volumenverschiebungen ist die Indikation für einen zentralvenösen Zugang zur Applikation von Vasoaktiva und Inotropika großzügig zu stellen. Gleiches gilt für den Einsatz der transösophagealen Echokardiografie (TEE) [10] ( Abb. 1), um den PAP und die Rechtsherzfunktion abzuschätzen und die Volumen- und katecholaminerge Therapie besser steuern zu können [18].

\section{INFO}

Pulmonalarterieller Katheter

Als Alternative zur TEE kann die Implementierung eines pulmonalarteriellen Katheters (PAK) erwogen werden. Es gibt zwar zahlreiche Studien, vorwiegend aus dem intensivmedizinischen Bereich, die dem PAK einen Nutzen zur Therapiesteuerung absprechen. Durch die Methode lassen sich jedoch Herzzeitvolumen, PVR und PAP ablesen und die gemischtvenöse Sauerstoffsättigung bestimmen. In der Konsequenz ist der PAK zur individuellen Volumentherapie, Applikation von Katecholaminen und medikamentösen Drucksenkung im kleinen Kreislauf hilfreich [19].

\section{Einfluss der maschinellen Beatmung}

Nach Einleitung der Anästhesie und mit Beginn der Beatmung ändern sich die intrathorakalen Druckverhältnisse. Tidalvolumen und positiv endexspiratorischer Druck bestimmen den intrathorakalen Druck, der die pulmonale und die systemische Zirkulation substanziell beeinflusst. Ein zu hoher intrathorakaler Druck komprimiert die pulmonalen Arteriolen und führt nachfolgend sowohl zur Erhöhung des PAP als auch zur Druckbelastung des rechten Ventrikels [20]. Auf der anderen Seite resultiert ein zu niedriges intrathorakales Lungenvolumen in der Ausbildung von Atelektasen. In diesen Arealen bildet sich eine hypoxisch pulmonale Vasokonstriktion, die ebenfalls den PAP steigert und die rechtsventrikuläre Nachlast erhöht.

\section{Merke}

In der klinischen Praxis sind Tidalvolumen und positiv endexspiratorischer Druck so zu titrieren, dass sich eine gute Oxygenierung, keine Azidose und ein niedrig-normaler $\mathrm{paCO}_{2}$ einstellen.

Der Beginn der maschinellen Beatmung bei der Anästhesieeinleitung stellt einen kritischen Moment bei Patienten mit PH dar: Nahezu alle gängigen Anästhetika vermindern den systemischen Widerstand. Sie können somit die (rechts-)koronare Perfusion bei einem in der Regel hypertrophierten und dilatierten rechten Ventrikel kritisch senken. Diese Situation wird durch den Beginn der maschinellen Beatmung mit konsekutiver rechtsventrikulärer Nachlasterhöhung und rechtsatrialer Vorlastsenkung kompliziert und kann in eine Rechtsherzdysfunktion münden. Trotz der Applikation von Vasokonstriktoren zur Kompensation des systemischen Widerstandsverlusts während der Anästhesieeinleitung muss bei einer schweren $\mathrm{PH}$ dennoch mit einer akuten Rechtsherzdekompensation gerechnet werden.

\section{Narkoseführung intraoperativ}

\section{Einfluss der Anästhetika}

Zur Aufrechterhaltung der Narkose bietet sich eine balancierte oder auch totalintravenöse Anästhesie mit Propofol an ( $\triangleright$ Tab. 3). Die Überlegenheit eines Verfahrens ist nicht belegt. Alle modernen volatilen Anästhetika wirken konzentrationsabhängig negativ inotrop, vermindern den systemischen Widerstand und können in höherer Dosierung die hypoxisch pulmonale Vasokonstriktion beeinträchtigen. Eine reine Inhalationsanästhesie erscheint deshalb gegenüber einer balancierten Technik mit Opioiden weniger geeignet [21]. Auf Lachgas sollte aufgrund der pulmonal-vaskulären Widerstandserhöhung bei Patienten mit PH verzichtet werden [22].

\section{Einfluss chirurgischer Verfahren}

Bei Operationen mit dem Risiko pulmonaler Embolien z. B. von Fett, Zement oder Luft ist besondere Vorsicht geboten - denn diese können zur akuten pulmonalen Druckerhöhung und folglich Rechtsherzdekompensation führen.

\section{INFO}

\section{Laparoskopische Operationen}

Oftmals bestehen Bedenken zur Durchführung laparoskopischer Techniken bei Patienten mit PH. Den Vorteilen reduzierter chirurgischer Invasivität, geringerer postoperativer Schmerzen und schnellerer Rekonvaleszenz stehen die Nachteile einer $\mathrm{CO}_{2}$-Insufflation und intraabdominellen Druckerhöhung gegenüber. Das Pneumoperitoneum erhöht die linksventrikuläre Nachlast, die rechtsventrikuläre Vorlast, den $\mathrm{paCO}_{2}$ und den Atemwegsdruck [23]. Diese Veränderungen beeinflussen zwar die $\mathrm{PH}$ und die Rechtsherzdysfunktion negativ, dennoch existieren zahlreiche Fallberichte, die eine erfolgreiche Durchführung laparoskopischer Eingriffe bei Patienten mit PH beschreiben [24]. Nach Auffassung der Autoren sind essenzielle Voraussetzungen für die Durchführung laparoskopischer Operationen in dieser Patientengruppe

- die Aufrechterhaltung einer Normokapnie,

- ein genaues Monitoring und

- eine zuvor gemeinsam besprochene chirurgische „Exit-Strategie“, die einen unmittelbaren Abbruch der Laparoskopie ermöglicht. 


\section{Kreislaufunterstützung - Übersicht}

Eine systemische Hypotension und eine zunehmende pulmonale Vasokonstriktion sind häufige - und im schlimmsten Fall letal endende - Komplikationen bei Patienten mit PH [25]. Die 3 wichtigsten hämodynamischen Veränderungen, die während der perioperativen Versorgung von Patienten mit $\mathrm{PH}$ vermieden werden müssen, sind deshalb:

- ein Anstieg des PVR,

- ein Abfall der koronaren Perfusion sowie

- eine Rechtsherzdekompensation ( $\bullet$ Abb. 2).

Im Falle einer Verschlechterung müssen sie eine konsequente Behandlung erfahren [26].

\section{Merke}

Da die akut-therapeutischen Ansätze die Ursache der $\mathrm{PH}$ berücksichtigen sollten, ist präoperativ ein individueller perioperativer Behandlungsplan festzulegen.

\section{Behandlung der pulmonalen Hypertonie}

Neben Hypoxie, Hyperkapnie und Azidose konstringieren auch eine stressbedingte Katecholaminfreisetzung und eine Hypothermie die pulmonalen Gefäße, sodass Normothermie mittels Wärmemanagement und Stressvermeidung wichtig erscheint.

\section{Merke}

Grundsätzlich ist die inhalative Applikation pulmonaler Vasodilatatoren der i.v. Verabreichung vorzuziehen, da Letztere fast immer mit einer Erniedrigung des systemischen Widerstandes verbunden ist.

Zur medikamentösen Senkung eines erhöhten PAP stehen perioperativ die nachfolgend erläuterten Substanzen zur Verfügung ( $\triangleright$ Tab. 4).

\section{Prostazykline}

Prostazykline stimulieren die Adenylatzyklase, erhöhen dadurch die intrazelluläre Konzentration an zyklischem Adenosinmonophosphat (cAMP) und dilatieren die pulmonalen Gefäße [29]. Neben Epoprostenol und Treprostinil stellt das Prostazyklin-Analogon Iloprost wohl den bekanntesten Vertreter dieser Substanzgruppe dar. Ilo- prost kann sowohl i. v. als auch über ein Verneblersystem inhalativ appliziert werden [30]. Aufgrund der kurzen Halbwertszeit von 5-25 min erfolgt die Anwendung von Iloprost 6- bis 8-mal pro Tag. Das Medikament senkt wirkungsvoll den PVR, scheint nach Absetzen nur einen geringen Rebound-Effekt aufzuweisen und kann bei spontan atmenden Patienten eingesetzt werden [31,32]. Bei unzureichender Wirkung lassen sich durch die Kombination von lloprost mit inhalativem Stickstoffmonoxid additive Effekte erzielen [33].

\section{Inhalatives Stickstoffmonoxid}

Stickstoffmonoxid wirkt über die Erhöhung von zyklischem Guanosinmonophosphat (cGMP) an der glatten Gefäßmuskulatur vasodilatierend. Der Vorteil der inhalativen Applikation liegt in der spezifischen Wirkung auf pulmonale Gefäße. Bei Übertritt in das Kapillarsystem wird inhalatives Stickstoffmonoxid (iNO) durch Bindung an Hämoglobin inaktiviert, sodass systemische Nebenwirkungen weitgehend ausbleiben. Aus diesem Umstand erklärt sich auch die kurze Halbwertszeit des Gases, die eine kontinuierliche Applikationsform bedingt. Nicht alle Patienten profitieren im Sinne einer Drucksenkung von iNO. Einige reagieren nicht oder nur unzureichend („Nonresponder“), zudem können bei Absetzen von iNO Rebound-Phänomene mit Erhöhung des PVR auftreten [34, 35].

\section{Phosphodiesterase-5-Inhibitoren}

Wie iNO entfalten die Phosphodiesterase-5-Inhibitoren ihre Wirkung über den cGMP-Weg, blockieren hierbei deren Abbau und führen zu einer Vasodilatation. Neben Tadalafil und Riociguat ist insbesondere Sildenafil bekannt und wird zur Behandlung der PH eingesetzt. Trotz des intravenösen oder oralen Applikationsweges wirken Phosphodiesterase-5-Inhibitoren aufgrund der Zielrezeptorverteilung vorwiegend in der Lunge. Der vasodilatierende Effekt ist dadurch in der pulmonalen Strombahn ausgeprägter als in der systemischen Zirkulation, dennoch ist bei bereits bestehender Hypotension Vorsicht geboten $[27,36]$. Mit der Gabe von Sildenafil lässt sich auch der unerwünschte Rebound-Effekt bei Entwöhnung einer iNO-Therapie vermindern [37].

D Tab.4 Pulmonal vasodilatierende Medikamente für den perioperativen Einsatz $[10,17,27,28]$.

\begin{tabular}{|c|c|c|c|c|}
\hline Substanz & Administration & Dosierung & PVR & SVR \\
\hline Stickstoffmonoxid (NO) & inhalativ & 20-80 ppm & $\downarrow$ & 0 \\
\hline Iloprost & inhalativ & $20-25 \mu \mathrm{g}$ & $\downarrow$ & 0 \\
\hline Iloprost & i.v. & $1,25-2,5 \mathrm{ng} / \mathrm{kg} \mathrm{KG} / \mathrm{min}$ & $\downarrow$ & $\downarrow$ \\
\hline Sildenafil & p.o. & $3 \times 20 \mathrm{mg}$ & $\downarrow$ & $0-\downarrow$ \\
\hline Sildenafil & i.v. & $10 \mathrm{mg}$ & $\downarrow$ & $0-\downarrow$ \\
\hline
\end{tabular}




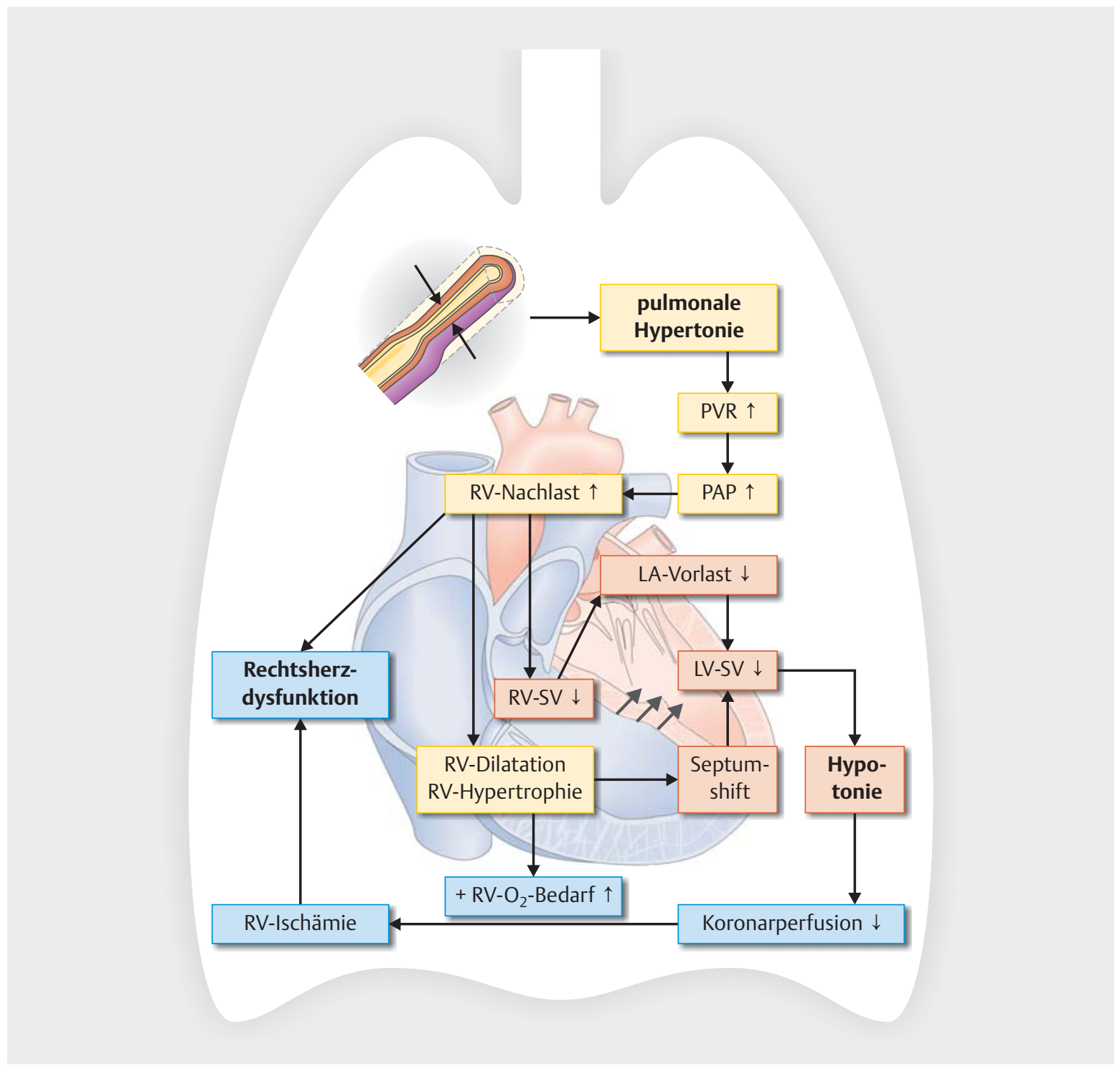

- Abb. 2 Schematische Darstellung der Interaktion von präkapillärer pulmonaler Hypertonie und Rechtsherzfunktion [26]. RA: rechter Vorhof, LA: linker Vorhof, LV: linker Ventrikel, RV: rechter Ventrikel, PAP: pumonalarterieller Druck, PVR: pulmonal-vaskulärer Widerstand, SV: Schlagvolumen, $\uparrow:$ Zunahme, $\downarrow:$ Abnahme.

Endothelin-Rezeptor-Antagonisten

\section{und Kalziumkanalblocker}

Endothelin-Rezeptor-Antagonisten eignen sich nicht zur akuten perioperativen Therapie. Sie zu kennen ist dennoch vorteilhaft, zumal diese Substanzgruppe bei Patienten mit $\mathrm{PH}$ regelhaft in der Begleitmedikation zu finden ist. Endothelin-A- und -B-Rezeptoren vermitteln eine pulmonale Vasokonstriktion. Bosentan, Ambrisentan oder Macitentan sind typische Vertreter der Endothelin-Rezeptor-Antagonisten. Sie werden bis zum gewünschten Plasmaspiegel über Wochen in ihrer Dosis erhöht und zeigen neben der Verminderung des PVR antiproliferative und antifibrotische Effekte [38].
Unter bestimmten Bedingungen werden Kalziumkanalblocker bei Patienten mit PH als Dauertherapie eingesetzt [10]. Der große Nachteil zeigt sich in der unselektiven Vasodilatation, die perioperativ mit einer erheblichen systemischen Hypotonie einhergehen kann. Aufgrund dieses Nebenwirkungsspektrums und den oben aufgezeigten Alternativen empfehlen die Autoren, auf Kalziumantagonisten zur Senkung des PVR intraoperativ zu verzichten.

\section{Behandlung einer Rechtsherzdysfunktion}

Durch die stetige Druckbelastung des rechten Herzens liegt - abhängig von der Dauer und dem Schweregrad der PH - oftmals eine Rechtsherzhypertrophie, -dilata- 
- Tab. 5 Vasokonstringierende und positiv inotrope Medikamente für den perioperativen Einsatz [10, 17, 39].

\begin{tabular}{|c|c|c|c|c|c|}
\hline Substanz & Administration & Dosierung & PVR & SVR & Kontraktilität \\
\hline Noradrenalin & kontinuierlich i.v & $0,03-0,25 \mu \mathrm{g} / \mathrm{kg} \mathrm{KG} / \mathrm{min}$ & $\uparrow$ & $\uparrow$ & 0 \\
\hline Vasopressin & kontinuierlich i.v. & $0,01-0,03 \mathrm{U} / \mathrm{kg} \mathrm{KG} / \mathrm{h}$ & 0 & $\uparrow$ & 0 \\
\hline Adrenalin & kontinuierlich i.v. & $0,05(-2) \mu \mathrm{g} / \mathrm{kg} \mathrm{KG} / \mathrm{min}$ & $\downarrow$ & $\uparrow$ & $\uparrow$ \\
\hline Dobutamin & kontinuierlich i.v. & $2-10 \mu \mathrm{g} / \mathrm{kg} \mathrm{KG} / \mathrm{min}$ & $\downarrow$ & $0-\downarrow$ & $\uparrow$ \\
\hline Milrinon & i.v. & $0,375-0,75 \mu \mathrm{g} / \mathrm{kg} \mathrm{KG} / \mathrm{min}$ & $\downarrow$ & $\downarrow$ & $\uparrow$ \\
\hline Milrinon & inhalativ & $1-5 \mathrm{mg}$ & $\downarrow$ & 0 & 0 \\
\hline Levosimendan & i.v. & $(0,05)-0,1-(0,2) \mu \mathrm{g} / \mathrm{kg} \mathrm{KG} / \mathrm{min}$ & $\downarrow$ & $\downarrow$ & $\uparrow$ \\
\hline
\end{tabular}

tion und -dysfunktion vor [26]. Ziele einer Unterstützung des rechten Herzens sind neben der o.g. Senkung des pulmonalarteriellen Drucks, eine ausreichende (rechts-) koronare Perfusion und rechtsventrikuläre Auswurfleistung zu garantieren ( $\boldsymbol{A} \mathbf{A} \boldsymbol{b} \boldsymbol{b} . \mathbf{2}$ ). Hierfür stehen folgende Substanzgruppen zur Verfügung ( $\triangleright$ Tab. 5).

\section{Adrenorezeptor- und Vasopressinrezeptor-Agonisten}

Um den nutritiven Blutfluss für den vorgeschädigten rechten Ventrikel aufrechtzuerhalten und eine regionale Sauerstoffunterversorgung zu vermeiden, zeigt die kontinuierliche Gabe des Alpha ${ }_{1}$-Rezeptoragonisten Noradrenalin eine gute Steuerbarkeit [17]. Nach Ansicht der Autoren sollte bei Patienten mit schwerer PH die Applikation des Vasokonstriktors bereits vor Einleitung der Anästhesie unter direkter arterieller Blutdrucküberwachung begonnen werden. So kann der zu antizipierende Blutdruckabfall bei der Einleitung vermieden werden.

Die Dosierung sollte titrierend erfolgen, da als Nebenwirkung von Noradrenalin die Widerstandserhöhung im kleinen Kreislauf bekannt ist. Ist dies der Fall, kann zusätzlich oder alternativ Vasopressin zur Anwendung kommen. Auch wenn verlässliche klinische Daten bei Patienten mit PH fehlen, demonstrieren Fallberichte und kleinere Studien bei Kindern mit PH und herzchirurgischen Patienten: Vasopressin erhöht - über den V1-Rezeptor vermittelt den systemischen Widerstand und beeinflusst den PVR wenig $[40,41]$.

\section{Merke \\ Nach den eigenen Erfahrungen der Autoren lassen sich Vasopressin und Noradrenalin kombinieren und die Wirkungen beider Vasokonstriktiva gut steuern.}

Über die Stimulation von Beta-Rezeptoren erhöhen die Substanzen Adrenalin und Dobutamin das intrazelluläre cAMP. Ihren dosisabhängigen Wirkprofilen entsprechend führen sie zu einer Zunahme von Kontraktilität, Herzzeitvolumen und nachfolgend systemischem sowie pulmo- nalem Blutfluss. Ungünstig bleibt die damit assoziierte Tachykardie, die den kardialen Sauerstoffbedarf erhöht [39].

\section{Phosphodiesterase-3-Inhibitoren}

Phosphodiesterase-3-Inhibitoren zeigen in der Behandlung einer Rechtsherzinsuffizienz bei bestehender $\mathrm{PH}$ ein günstiges Wirkspektrum. Durch Hemmung des CAMPAbbaus steigern Phosphodiesterase-3-Inhibitoren zum einen die kardiale Kontraktilität und vermindern gleichzeitig den pulmonalen Gefäßtonus. Nachteilig ist die damit verbundene systemische Widerstandsabnahme, die durch Vasokonstriktoren kompensiert werden muss. Für Milrinon, den bekanntesten Vertreter der Phosphodiesterase-3-Inhibitoren, stehen eine i.v. sowie eine inhalative Applikationsform zur Verfügung ( $\bullet$ Tab.5). Letztere führt zu einer selektiven pulmonalen Vasodilatation ohne wesentliche systemische Nebenwirkungen [42].

\section{Kalzium-Sensitizer}

Levosimendan erhöht die Kalziumsensitivität kontraktiler Proteine und hemmt gleichzeitig die Phosphodiesterase3. Daraus resultieren als Wirkungen eine Zunahme der Herzkraft und des Herzzeitvolumens sowie eine Vasodilatation mit Verminderung des PVR. Die rechtsventrikuläre Funktion lässt sich durch diese Kombination verbessern $[43,44]$. Im Gegensatz zu den Betamimetika ändern Kalzium-Sensitizer nichts am myokardialen Sauerstoffverbrauch. Nachteilig sind zum einen die oft begleitende systemische Hypotonie und zum anderen die derzeit hohen Therapiekosten.

\section{Schwangere Patientinnen mit pulmonaler Hypertonie}

Patientinnen mit schwerer $\mathrm{PH}$ wird nach wie vor von einer Schwangerschaft abgeraten, da die peripartale Letalität bei schwerer $\mathrm{PH}$ geschätzt bei $20 \%$ liegt $[45,46]$. Als Haupttodesursache ist trotz moderner Behandlungskonzepte das akute Rechtsherzversagen führend [10]. Die Mehrheit geburtshilflicher Zentren bevorzugt bei diesem 
- Tab. 6 Entscheidungshilfen zur postoperativen Betreuungssituation.

\begin{tabular}{|l|l|}
\hline Aufwachraum & Intensivstation \\
\hline - Patienten der funktionellen & - Patienten der funktionellen \\
Klasse 1-2 & Klasse 3-4 \\
\hline " "kleiner“ Eingriff & " "großer“ Eingriff \\
\hline - geringe Schmerzen & - Hypothermie \\
\hline - stabile Hämodynamik & - erhebliche Schmerzen \\
\hline - gute Oxygenierung und Ventila- & - Einschränkung in der Atmung \\
tion & - Katecholamintherapie \\
& - zu erwartende Volumen- \\
& verschiebungen \\
& \\
\hline
\end{tabular}

Patientenkollektiv eine Entbindung per Sectio caesarea. Bezüglich des Narkoseverfahrens existieren keine verlässlichen Daten oder klare Empfehlungen. Die oben genannten Aspekte zum invasiven Monitoring, der Behandlung der $\mathrm{PH}$ und der Rechtsherzdysfunktion gelten in gleichem Maße bei schwangeren Patientinnen. Außer den teratogen wirkenden Endothelin-Rezeptor-Antagonisten [47] können die pulmonalen Vasodilatatoren iNO, Iloprost und Sildenafil zum Einsatz kommen.

\section{Postoperativ}

Je schwerer die Erkrankung und je ausgeprägter die chirurgische Intervention, desto wichtiger ist in der postoperativen Phase eine intensivmedizinische Betreuung mit erfahrenem Personal ( $\bullet$ Tab. 6).

Klare Empfehlungen, welche Patienten im Aufwachraum betreut werden können und welche einer Verlegung auf die Intensivstation bedürfen, gibt es nicht.

\section{Merke}

Zu bedenken ist, dass ein erheblicher Anteil der perioperativen Komplikationen bei den Patienten mit PH postoperativ auftritt.

Eine Ursache stellt die fehlende Vigilanz für eine sich möglicherweise innerhalb von Minuten entwickelnde Dekompensation dar [48]. Als Auslöser einer akuten Rechtsherzinsuffizienz lassen sich Hypoventilation, Hypoxie, sympathische Reaktionen auf Schmerzen, Flüssigkeitsverschiebungen oder Arrhythmien beispielhaft benennen [12].

\section{KERNAUSSAGEN}

- Die präoperative Evaluation und Planung des Patienten mit pulmonaler Hypertonie sind für die sichere Durchführung der Anästhesie essenziell.

- Hypoxie, Hyperkapnie, Azidose, Hypothermie, Stress und Blutdruckabfall gilt es strikt zu vermeiden.

- Die Beatmung sollte so wenig invasiv wie möglich eingestellt und der PEEP titriert werden.

- Zur Senkung des pulmonalarteriellen Drucks eignen sich vor allem inhalativ applizierte pulmonale Vasodilatatoren wie Stickstoffmonoxid oder Prostazykline bzw. deren Kombination.

- Bei i.v. verabreichten pulmonalen Vasodilatatoren wie Phosphodiesterase-3- und Phosphodiesterase5-Inhibitoren muss ein begleitender Abfall des Blutdrucks berücksichtigt und konsequent behandelt werden.

- Bei rechtsventrikulärer Dysfunktion gilt es, mittels inotroper Substanzen und Inodilatoren die Herzfunktion zu unterstützen.

- Auch in der postoperativen Phase besteht ein erhebliches Risiko der Dekompensation des Krankheitsbildes und es bedarf einer intensiven Überwachung.

\section{Interessenkonflikt}

Die Autoren geben an, dass kein Interessenkonflikt besteht.

Autorinnen/Autoren

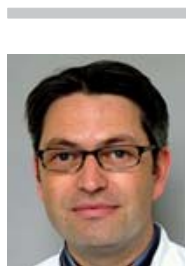

\section{Alexander Hötzel}

Prof. Dr. med., Medizinstudium an der Universität Freiburg. Facharztausbildung und Postdoctoral Fellow an der Klinik für Anästhesiologie und Intensivmedizin Freiburg. 2005-2007 DFG-Stipendiat am University of Pittsburgh Medical Center. 2008-2010 Masterstudium Gesundheitsmanagement an der TU Kaiserslautern und Universität Witten/Herdecke. Seit 2010 Geschäftsführender Oberarzt der Klinik für Anästhesiologie und Intensivmedizin, Uniklinik Freiburg.

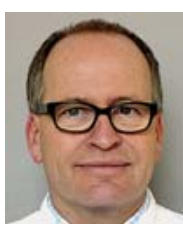

\section{Torsten Loop}

Prof. Dr. med., Medizinstudium an der Universität Freiburg. 1991-1992 Assistenzarzt Kantonsspital Liestal/Schweiz. 1992-1995 Wissenschaftlicher Assistent an der Anästhesiologischen Universitätsklinik Freiburg. Seit 1996 Facharzt an der Anästhesiologischen Uniklinik Freiburg. 2006 Habilitation, 2010 außerplanmäßige Professur. Seit 2011 Leitender Oberarzt der Klinik für Anästhesiologie und Intensivmedizin, Uniklinik Freiburg. 


\section{Korrespondenzadresse}

\section{Prof. Dr. Alexander Hötzel}

Klinik für Anästhesiologie und Intensivmedizin

Universitätsklinikum Freiburg

Hugstetter Straße 55

79106 Freiburg

alexander.hoetzel@uniklinik-freiburg.de

\section{Wissenschaftlich verantwortlich} gemäß Zertifizierungsbestimmungen

Wissenschaftlich verantwortlich gemäß Zertifizierungsbestimmungen für diesen Beitrag ist Prof. Dr. med. Alexander Hötzel, Freiburg.

\section{Literatur}

[1] Luecke E, Schmeißer A, Schreiber J. Pulmonale Hypertonie. Anasthesiol Intensivmed Notfallmed Schmerzther 2019; 54; 320-333

[2] Hoeper MM, Bogaard HJ, Condliffe R et al. Definitions and diagnosis of pulmonary hypertension. J Am Coll Cardiol 2013; 62: D42-D50

[3] Galie N, Humbert M, Vachiery JL et al. 2015 ESC/ERS Guidelines for the diagnosis and treatment of pulmonary hypertension: The Joint Task Force for the Diagnosis and Treatment of Pulmonary Hypertension of the European Society of Cardiology (ESC) and the European Respiratory Society (ERS): Endorsed by: Association for European Paediatric and Congenital Cardiology (AEPC), International Society for Heart and Lung Transplantation (ISHLT). Eur Heart J 2016; 37: 67-119

[4] Fang JC, DeMarco T, Givertz MM et al. World Health Organization Pulmonary Hypertension group 2: pulmonary hypertension due to left heart disease in the adult - a summary statement from the Pulmonary Hypertension Council of the International Society for Heart and Lung Transplantation. J Heart Lung Transplant 2012; 31: 913-933

[5] Taichman DB, Ornelas ], Chung L et al. Pharmacologic therapy for pulmonary arterial hypertension in adults: CHEST guideline and expert panel report. Chest 2014; 146: 449-475

[6] Hoeper MM, Ghofrani HA, Gorenflo M et al. Diagnostik und Therapie der pulmonalen Hypertonie: Europäische Leitlinien 2009. Pneumologie 2010; 64: 401-414

[7] Benza RL, Miller DP, Gomberg-Maitland M et al. Predicting survival in pulmonary arterial hypertension: insights from the Registry to Evaluate Early and Long-Term Pulmonary Arterial Hypertension Disease Management (REVEAL). Circulation 2010; 122: 164-172

[8] Galderisi M, Cosyns B, Edvardsen T et al. Standardization of adult transthoracic echocardiography reporting in agreement with recent chamber quantification, diastolic function, and heart valve disease recommendations: an expert consensus document of the European Association of Cardiovascular Imaging. Eur Heart J Cardiovasc Imaging 2017; 18: 13011310

[9] Yang El. Perioperative management of patients with pulmonary hypertension for non-cardiac surgery. Curr Rheumatol Rep 2015; 17: 15

[10] Pilkington SA, Taboada D, Martinez G. Pulmonary hypertension and its management in patients undergoing non-cardiac surgery. Anaesthesia 2015; 70: 56-70
[11] Divers C, Platt D, Wang E et al. A review of clinical trial endpoints of patients with pulmonary arterial hypertension and chronic thromboembolic pulmonary hypertension and how they relate to patient outcomes in the United States. J Manag Care Spec Pharm 2017; 23: 92-104

[12] Ramakrishna G, Sprung J, Ravi BS et al. Impact of pulmonary hypertension on the outcomes of noncardiac surgery: predictors of perioperative morbidity and mortality. J Am Coll Cardiol 2005; 45: 1691-1699

[13] Missant C, Rex S, Claus P et al. Thoracic epidural anaesthesia disrupts the protective mechanism of homeometric autoregulation during right ventricular pressure overload by cardiac sympathetic blockade: a randomised controlled animal study. Eur J Anaesthesiol 2011; 28: 535-543

[14] Rex S, Missant C, Segers P et al. Thoracic epidural anesthesia impairs the hemodynamic response to acute pulmonary hypertension by deteriorating right ventricular-pulmonary arterial coupling. Crit Care Med 2007; 35: 222-229

[15] Fischer LG, Van Aken H, Burkle H. Management of pulmonary hypertension: physiological and pharmacological considerations for anesthesiologists. Anesth Analg 2003; 96: 1603 1616

[16] Kaye AD, Baluch A, Phelps ] et al. An analysis of remifentanil in the pulmonary vascular bed of the cat. Anesth Analg 2006; 102: $118-123$

[17] Preckel B, Eberl S, Frassdorf J et al. Management von Patienten mit pulmonaler Hypertonie. Anaesthesist 2012; 61: 574-577

[18] Schulmeyer MC, Santelices E, Vega R et al. Impact of intraoperative transesophageal echocardiography during noncardiac surgery. J Cardiothorac Vasc Anesth 2006; 20: 768-771

[19] McLaughlin VV, Presberg KW, Doyle RL et al. Prognosis of pulmonary arterial hypertension: ACCP evidence-based clinical practice guidelines. Chest 2004; 126: 78S-92S

[20] Strumpher J, Jacobsohn E. Pulmonary hypertension and right ventricular dysfunction: physiology and perioperative management. J Cardiothorac Vasc Anesth 2011; 25: 687-704

[21] Fox C, Kalarickal PL, Yarborough M] et al. Perioperative management including new pharmacological vistas for patients with pulmonary hypertension for noncardiac surgery. Curr Opin Anaesthesiol 2008; 21: 467-472

[22] Schulte-Sasse U, Hess W, Tarnow J. Pulmonary vascular responses to nitrous oxide in patients with normal and high pulmonary vascular resistance. Anesthesiology 1982; 57: 9-13

[23] Atkinson TM, Giraud GD, Togioka BM et al. Cardiovascular and ventilatory consequences of laparoscopic surgery. Circulation 2017; 135: 700-710

[24] Montandrau O, Mascitti P, Boucau C et al. Laparoscopic rectopexy in a patient with pulmonary hypertension associated with scleroderma: a case report. A A Pract 2018; doi:10.1213/ XAA.0000000000000919

[25] Tonelli AR, Arelli V, Minai OA et al. Causes and circumstances of death in pulmonary arterial hypertension. Am J Respir Crit Care Med 2013; 188: 365-369

[26] Cheng JW, Tonelli AR, Pettersson G et al. Pharmacologic management of perioperative pulmonary hypertension. J Cardiovasc Pharmacol 2014; 63: 375-384

[27] Vachiery JL, Huez S, Gillies H et al. Safety, tolerability and pharmacokinetics of an intravenous bolus of sildenafil in patients with pulmonary arterial hypertension. $\mathrm{Br}$ J Clin Pharmacol 2011; 71: 289-292

[28] Thunberg CA, Morozowich ST, Ramakrishna H. Inhaled therapy for the management of perioperative pulmonary hypertension. Ann Card Anaesth 2015; 18: 394-402 
[29] Mubarak KK. A review of prostaglandin analogs in the management of patients with pulmonary arterial hypertension. Respir Med 2010; 104: 9-21

[30] Tissot C, Beghetti M. Review of inhaled iloprost for the control of pulmonary artery hypertension in children. Vasc Health Risk Manag 2009; 5: 325-331

[31] Rex S, Schaelte G, Metzelder S et al. Inhaled iloprost to control pulmonary artery hypertension in patients undergoing mitral valve surgery: a prospective, randomized-controlled trial. Acta Anaesthesiol Scand 2008; 52: 65-72

[32] Winterhalter M, Simon A, Fischer $S$ et al. Comparison of inhaled iloprost and nitric oxide in patients with pulmonary hypertension during weaning from cardiopulmonary bypass in cardiac surgery: a prospective randomized trial. J Cardiothorac Vasc Anesth 2008; 22: 406-413

[33] Antoniou T, Koletsis EN, Prokakis C et al. Hemodynamic effects of combination therapy with inhaled nitric oxide and iloprost in patients with pulmonary hypertension and right ventricular dysfunction after high-risk cardiac surgery. J Cardiothorac Vasc Anesth 2013; 27: 459-466

[34] Griffiths M], Evans TW. Inhaled nitric oxide therapy in adults. N Engl J Med 2005; 353: 2683-2695

[35] Hurford WE, Bigatello LM. NO-body's perfect. Anesthesiology 2002; 96: 1285-1287

[36] Buckley MS, Staib RL, Wicks LM et al. Phosphodiesterase-5 inhibitors in management of pulmonary hypertension: safety, tolerability, and efficacy. Drug Healthc Patient Saf 2010; 2: 151-161

[37] Lee JE, Hillier SC, Knoderer CA. Use of sildenafil to facilitate weaning from inhaled nitric oxide in children with pulmonary hypertension following surgery for congenital heart disease. J Intensive Care Med 2008; 23: 329-334

[38] Channick RN, Simonneau G, Sitbon O et al. Effects of the dual endothelin-receptor antagonist bosentan in patients with pulmonary hypertension: a randomised placebo-controlled study. Lancet 2001; 358: 1119-1123

[39] Riessen R, Tschritter O, Janssens U et al. Katecholamine: Pro und Contra. Med Klin Intensivmed Notfmed 2016; 111: 37-46
[40] Mizota T, Fujiwara K, Hamada M et al. Effect of arginine vasopressin on systemic and pulmonary arterial pressure in a patient with pulmonary hypertension secondary to pulmonary emphysema: a case report. JA Clin Rep 2017; 3: 1

[41] Tayama E, Ueda T, Shojima T et al. Arginine vasopressin is an ideal drug after cardiac surgery for the management of low systemic vascular resistant hypotension concomitant with pulmonary hypertension. Interact Cardiovasc Thorac Surg 2007; 6: 715-719

[42] Wang H, Gong M, Zhou B et al. Comparison of inhaled and intravenous milrinone in patients with pulmonary hypertension undergoing mitral valve surgery. Adv Ther 2009; 26: 462-468

[43] Russ MA, Prondzinsky R, Carter JM et al. Right ventricular function in myocardial infarction complicated by cardiogenic shock: Improvement with levosimendan. Crit Care Med 2009; 37: 3017-3023

[44] Papp Z, Edes I, Fruhwald S et al. Levosimendan: molecular mechanisms and clinical implications: consensus of experts on the mechanisms of action of levosimendan. Int J Cardiol 2012; 159: 82-87

[45] Jais X, Olsson KM, Barbera JA et al. Pregnancy outcomes in pulmonary arterial hypertension in the modern management era. Eur Respir J 2012; 40: 881-885

[46] Rosengarten D, Blieden LC, Kramer MR. Pregnancy outcomes in pulmonary arterial hypertension in the modern management era. Eur Respir J 2012; 40: 1304-1305

[47] Bassily-Marcus AM, Yuan C, Oropello J et al. Pulmonary hypertension in pregnancy: critical care management. Pulm Med 2012; 2012: 709407. doi:10.1155/2012/709407

[48] Hoeper MM, Granton J. Intensive care unit management of patients with severe pulmonary hypertension and right heart failure. Am J Respir Crit Care Med 2011; 184: 1114-1124

\section{Bibliografie}

DOI https://doi.org/10.1055/a-0668-4653

Anästhesiol Intensivmed Notfallmed Schmerzther 2019; 54: 334-346 @ Georg Thieme Verlag KG Stuttgart · New York ISSN 0939-2661 


\section{Punkte sammeln auf CME.thieme.de}

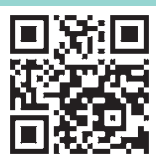

Diese Fortbildungseinheit ist in der Regel 12 Monate online für die Teilnahme verfügbar.

Den genauen Einsendeschluss finden Sie unter https://eref.thieme.de/CXBA4LJ.

Sollten Sie Fragen zur Online-Teilnahme haben, finden Sie unter https://cme.thieme.de/hilfe

eine ausführliche Anleitung. Wir wünschen viel Erfolg beim Beantworten

der Fragen!

Unter https://eref.thieme.de/CXBA4LJ oder über den QR-Code kommen Sie direkt zur Startseite des Wissenstests.

VNR 2760512019156641290

\section{Frage 1}

Welche Aussage zur Ursache einer pulmonalen Hypertonie (PH) ist richtig?

A Eine präkapilläre PH lässt sich von der postkapillären durch einen Röntgenthorax unterscheiden.

B Eine Aortenstenose führt klassischerweise zu einer präkapillären $\mathrm{PH}$.

C Die postkapilläre $\mathrm{PH}$ ist durch einen PAPm $\geq 25 \mathrm{mmHg}$ und einem PCWP $<15 \mathrm{mmHg}$ definiert.

D Die idiopathische pulmonalarterielle Hypertonie ist eine typische Ursache einer präkapillären $\mathrm{PH}$.

E Eine chronisch thromboembolische $\mathrm{PH}$ zählt zu den postkapillären Ursachen einer $\mathrm{PH}$.

\section{Frage 2}

Welche Aussage zur präoperativen Diagnostik der Patienten mit $\mathrm{PH}$ ist richtig?

A Ohne eine aktuelle Rechtsherzkatheter-Untersuchung sollten Patienten mit PH grundsätzlich nicht operiert werden.

B Vorliegende Befunde einer Rechtsherzkatheter-Untersuchung müssen anästhesiologisch objektiviert werden.

C Die Echokardiografie stellt den „Goldstandard“ zur Verifizierung einer $\mathrm{PH}$ dar.

D Die Durchführung einer Lungenfunktionsuntersuchung ist bei allen Patienten mit PH essenziell.

E Die Spirometrie muss bei allen Patienten mit PH durchgeführt werden.

\section{Frage 3}

Welche Aussage ist richtig? Im Rahmen der Beurteilung der Schwere der $\mathrm{PH}$...

A muss die funktionelle Kapazität des Patienten vom Anästhesisten eruiert werden.

B zeigt die funktionelle Klassifikation keine Assoziation mit dem Überleben auf.

$\mathrm{C}$ ist die alleinige Echokardiografie ausreichend.

$\mathrm{D}$ ist die alleinige Rechtsherzkatheter-Untersuchung ausreichend.

E sind Laboruntersuchungen von ProBNP und Troponin-T entscheidend.

\section{Frage 4}

Welche Aussage zu Medikamenten zur Induktion einer Anästhesie beim Patienten mit PH ist richtig?

A Es dürfen nur Opioide mit kurzer Halbwertszeit genutzt werden.

B Für Propofol besteht eine Kontraindikation.

C Eine Maskeneinleitung mit Lachgas erscheint besonders günstig.

D Barbiturate sind die Mittel der Wahl.

E Etomidat beeinflusst die rechtsventrikuläre Kontraktion am geringsten.

\section{Frage 5}

Welche Aussage zur Auswahl des Monitorings bei Patienten mit $\mathrm{PH}$ ist richtig?

A Patienten aller funktionellen Klassen sollten perioperativ mittels Echokardiografie überwacht werden.

B Patienten der funktionellen Klasse 1 sollten eine invasive arterielle Druckmessung erhalten.

C Patienten der funktionellen Klasse 2 sollten einen zentralvenösen Zugang erhalten.

D Patienten der funktionellen Klasse 3 profitieren immer von einem pulmonalarteriellen Katheter.

E Patienten der funktionellen Klasse 4 sollten bei großen Operationen zusätzlich mittels Echokardiografie und/oder pulmonalarteriellem Katheter überwacht werden.

\section{Frage 6}

Welche Aussage ist falsch? Die maschinelle Beatmung bei Patienten mit $\mathrm{PH}$...

A hat einen oft erheblichen Einfluss auf die Hämodynamik.

B erniedrigt die rechtsventrikuläre Nachlast und erhöht die linksventrikuläre Nachlast.

C erniedrigt die rechtsatriale Vor- und linksventrikuläre Nachlast.

D kann zur Rechtsherzdekompensation führen.

E sollte so wenig invasiv wie möglich sein.

\section{- Weitere Fragen auf der folgenden Seite...}




\section{Punkte sammeln auf CME.thieme.de}

Fortsetzung $\ldots$

\section{Frage 7}

Welche Aussage ist falsch? Um eine perioperative Widerstandszunahme (PVR) im kleinen Kreislauf zu vermeiden bzw. zu senken, ...

A gilt es, Stress, Hypoxie, Hyperkapnie und Azidose zu verhindern.

B sollten primär Endothelin-Rezeptor-Antagonisten eingesetzt werden.

C zeigt die inhalative Applikation von Stickstoffmonoxid oder Prostazyklinen eine selektive Wirkung auf die Pulmonalgefäße.

D können inhalatives Stickstoffmonoxid und Prostazykline kombiniert werden.

E bietet sich u. U. auch oral oder i.v. verabreichtes Sildenafil an.

\section{Frage 8}

Welche Aussage zur medikamentösen Unterstützung der rechtsventrikulären Funktion ist richtig?

A Entscheidend ist ein ausreichender systemischer Widerstand, um die rechtskoronare Perfusion zu erhalten.

B Noradrenalin darf erst nach der Einleitung und nach Eintritt einer Hypotension appliziert werden.

C Vasopressin erhöht den systemischen, aber vor allem den pulmonal-vaskulären Widerstand.

D Adrenalin und Dobutamin führen über die intrazelluläre Erhöhung von cGMP zu einer Bradykardie.

E Levosimendan wirkt positiv inotrop, erhöht allerdings deutlich den myokardialen Sauerstoffverbrauch.

\section{Frage 9}

Welche Aussage zu schwangeren Patientinnen mit schwerer PH ist richtig?

A Bei schwangeren Patientinnen mit schwerer PH ist aufgrund moderner Behandlungskonzepte die peripartale Letalität auf $2 \%$ zurückgegangen.

B Die Haupttodesursache in diesem Patientenkollektiv ist eine im Laufe der Schwangerschaft auftretende Linksherzinsuffizienz.

C Sildenafil ist bei schwangeren Patientinnen mit PH kontraindiziert.

D Die pulmonalen Vasodilatatoren iNO und Iloprost dürfen bei schwangeren Patientinnen mit PH eingesetzt werden.

E Mittel der Wahl für schwangere Patientinnen mit PH sind Endothelin-Rezeptor-Antagonisten.

\section{Frage 10}

Welche Aussage ist richtig? In der postoperativen Betreuung von Patienten mit PH...

A ist nach den aktuellen Leitlinien immer ein intensivmedizinischer Aufenthalt indiziert.

B ist bei modernen strukturellen Voraussetzungen die Präsenz von erfahrenem Personal zu vernachlässigen.

$\mathrm{C}$ ist die analgetikabedingte Hypoventilation von untergeordneter Bedeutung.

D ist die Inzidenz von Komplikationen gering bzw. entwickeln sich diese in aller Regel über einen längeren Zeitraum.

E ist die Inzidenz von Komplikationen hoch und sie entwickeln sich oftmals innerhalb von Minuten. 